\title{
Quantity, Quality, or Neither-Measuring the Effectiveness of Rounds
}

\author{
Melissa A Plesac, MD*; Andrew PJ Olson, MD ${ }^{1,2}$
}

${ }^{1}$ Department of Medicine University of Minnesota Medical School, Minneapolis, Minnesota; ${ }^{2}$ Department of Pediatrics, University of Minnesota Medical School, Minneapolis, Minnesota.

M

edicine has a rich history of attending-led rounds, with some iteration of this ritual occurring as far back as the 1600s. In the early 1900s, the concept of "bedside rounds" was popularized by William Osler, who widely espoused their importance as a clinical and educational tool. Despite our best intentions, however, rounds today may be little reminiscent of the rounds of Osler's day. Recent investigations into the characteristics of rounds have specifically revealed a "shift in the format from the beside to conference rooms and hallways." 2 Most of our practices for rounding in the modern era are built on tradition and belief rather than evidence. The ecosystem of modern hospital care is dramatically different than that of Osler's day, and fundamental questions about the format, content, stakeholders, and processes of rounds remain. Perhaps the greatest and most needed change in rounding in recent years is the shift of rounds from a physician-centric activity to an activity that values the modern interprofessional hospital team. Ultimately, the very definition of "rounds" and the purpose they are meant to serve in the context of a dynamic and complicated hospital ecosystem has become increasingly complex and thus, difficult to assess and improve.

In this month's Journal of Hospital Medicine, Sang et al. ${ }^{3}$ address this complexity by returning to basics and utilizing a novel approach to precisely measure the frequency and duration of a necessary (albeit insufficient) condition for interdisciplinary bedside rounding to occur: colocation of physician, nurse, and patient. Ultimately, their results provide a springboard to ask more complex and meaningful questions. Why, despite a recent culture shift prioritizing a return to bedside, is substantive physician and nurse colocation so persistently difficult to attain? How can we study outcomes of interdisciplinary bedside rounds if we cannot reliably facilitate their occurrence? What does "effective" rounding even mean? That is, what variables would be both meaningful and sensitive to changes in rounds?

After centuries of rounding, the medical community would be presumed to have perfected this art; however, we are instead left with more questions than answers. Prior research efforts have demonstrated the shifting of rounds away from the bedside, with bedside rounds occurring only $10 \%-40 \%$ of the time based on bias-prone survey data. ${ }^{2,4}$ Interestingly, a study by Huang et al., designed specifically to increase implementation

*Corresponding Author: Melissa A Plesac, MD; E-mail: plesac01@umn.edu; Telephone: 219-741-1064; Twitter: @MelissaPlesacMD

Received: May 15, 2019; Revised: May 31, 2019; Accepted: June 3, 2019

(๔) 2019 Society of Hospital Medicine DOI 10.12788/jhm.3261 of interdisciplinary bedside rounds, showed a frequency of only $64 \%{ }^{5}$ These studies are focused primarily on parameters such as patient and nursing satisfaction and did not include other important outcomes such as length of stay, readmission rates, diagnostic quality, patient engagement, or mortality. ${ }^{2,46}$

In Sang et al., ${ }^{3}$ the authors utilized a real-time locator system, namely, radiofrequency identification, to precisely track the physical workflow of both attending hospitalists and bedside nurses and then subsequently used the data obtained to measure the frequency and duration of colocation at the patient bedside. The authors defined a physician "rounding event" as the physician's presence in a single bed patient room for at least 10 seconds. The study revealed that colocation of physician and nurse (for at least 10 seconds) occurred in only $30 \%$ of all physician rounding events recorded. The duration of a physician rounding event was 5.68 minutes without nurse colocation and 9.56 minutes if a nurse was present. No difference in the frequency of physician-nurse overlap was observed between weekdays and weekends. Interestingly and not surprisingly, patient rooms located farther from the nursing station had a decreased likelihood of physician-nurse overlap.

A greater understanding of the medical community's inability to reliably implement interdisciplinary bedside rounding may be found by examining the ecosystem of inpatient medicine. Physicians and nurses function in an environment with increasingly complex patients, more stringent (and nonevidence-based) documentation requirements, the physical decoupling of patients and their clinical information, and, as Sang et al. ${ }^{3}$ illuminate, complex geographical ward structures. As the rapidity with which patients are diagnosed and treated continues to escalate, physicians and nurses are also asked to attempt to squeeze an Oslerian-type rounding system into an ecosystem that is in overdrive. That the puzzle pieces do not fit should not be a surprise.

There is a risk that systems may implement interventions to "check the box" for interdisciplinary bedside rounding instead of seeking to change outcomes. How much time is time enough together at the bedside? Sang et al., among others, ponder whether a rounding duration of just under 10 minutes is enough. 3,6 However, Rothberg et al. demonstrated that increased duration of communication alone is not necessarily associated with increased patient satisfaction or nurse-physician agreement on plan of care, suggesting that colocation and communication are necessary but not sufficient for true interdisciplinary patient care. The discordance between communication and understanding can potentially be explained by the varying agendas of the members of the interdisciplinary team during the same interaction. ${ }^{8}$ 
Ultimately, the future of interdisciplinary bedside rounding, and rounding in general, remains uncertain. Potential areas for improvement and further study include patient regionalization, ${ }^{3,5}$ tools to align agendas among stakeholders, ${ }^{8}$ integrating recommendations for interdisciplinary communication,? and utilizing a common definition and taxonomy for study design..$^{10}$ These interventions may improve future study designs and outcomes. However, these interventions are small tweaks in a complex ecosystem, and the return on these interventions may eventually reach an asymptote. Perhaps the concept of rounding as we know it is broken beyond repair, and a more radical approach is needed: either the creation of a completely innovative shared mental model of acute care that acknowledges the complex environment of inpatient medicine, or a complete restructuring of the ecosystem itself. Nonetheless, the findings of Sang et al. ${ }^{3}$ with respect to the ongoing difficulty of implementing interdisciplinary bedside rounding elucidate the need for innovation in study design and rounding implementation strategies; they also prompt us to ask-and answer-the complicated questions related to this integral component of our practice.

Disclosures: The authors have nothing to disclose.

\section{References}

1. Linfors EW, Neelon FA. The case for bedside rounds. N Engl J Med. 1980;303(21):1230-1233. https://doi.org/10.1056/NEJM198011203032110.

2. Stickrath $C$, Noble M, Prochazka A, et al. Attending rounds in the current era. JAMA Intern Med. 2013;173(12):1084. https://doi.org/10.1001/jamainternmed.2013.6041.

3. Sang AX, Tisdale RL, Nielson D, et al. How much time are physicians and nurses spending together at the patient bedside? J Hosp Med. 2019;14(8):468473. https://doi.org/10.12788/jhm.3204.

4. O'leary KJ, Killarney A, Hansen LO, et al. Effect of patient-centred bedside rounds on hospitalised patients' decision control, activation and satisfaction with care. BMJ Qual Saf. 2016;25(12):921-928. https://doi.org/10.1136/bmjas-2015-005035.

5. Huang KTL, Minahan J, Brita-Rossi $P$, et al. All together now: impact of a regionalization and bedside rounding initiative on the efficiency and inclusiveness of clinical rounds OR. J Hosp Med. 2017;12(3):150-156. https://doi. org/10.12788/jhm.2696.

6. Gonzalo JD, Wolpaw DR, Lehman E, Chuang CH. Patient-centered interprofessional collaborative care: factors associated with bedside interprofessional rounds. J Gen Intern Med. 2014;29(7):1040-1047. https://doi.org/10.1007/ s11606-014-2817-x.

7. Rothberg MB, Steele JR, Wheeler J, Arora A, Priya A, Lindenauer PK. The relationship between time spent communicating and communication outcomes on a hospital medicine service. J Gen Intern Med. 2012;27(2):185-189. https://doi.org/10.1007/s11606-011-1857-8.

8. Holton R, Patel R, Eggebrecht M, et al. Rounding on rounds. Am J Med Qual. 2015;30(5):493-493. https://doi.org/10.1177/1062860615596388

9. Kassutto S, Seam N, Carlos WG, et al. Twelve tips for conducting successful interprofessional teaching rounds [published online ahead of print February 1, 2019]. Med Teach. https://doi.org/10.1080/0142159X.2018.1545086.

10. Bhamidipati VS, Elliott DJ, Justice EM, Belleh E, Sonnad SS, Robinson EJ. Structure and outcomes of interdisciplinary rounds in hospitalized medicine patients: a systematic review and suggested taxonomy. J Hosp Med. 2016;11(7):513-523. https://doi.org/10.1002/jhm.2575. 